Final Technical Report

DOE Award Number: DE-FG02-03ER25578

Project Title: A Level-of-Detail Approach to Cluster-based Visualization

Principle Investigator: Jonathan Cohen, Johns Hopkins University (now at LLNL)

\title{
Summary
}

Interaction has come to play an essential role in scientific visualization. It allows a scientist to explore large quantities of data derived from various sensor technologies as well as the output of complex simulations. These data are large enough to easily overwhelm even the most powerful graphics hardware available, making useful interaction with the data all but impossible.

One approach to achieving interaction is to trade fidelity for speed by employing multiresolution forms of the visualization data. This ability to control the level of detail can bring many visualization applications from a state of non-interactive performance to one of interactive performance. However, if the data are complex enough, the quality may be reduced beneath the threshold of useful visualization during periods of interaction.

Another classic approach to improving performance is to build a faster machine. 3D rendering is a highly parallelizable problem, so a number of large-scale parallel machines have been built over the years to push the state of the art, allowing the visualization of larger data sets. Today's parallel machines often take the form of networked clusters of PCs, built mostly from commodity parts. The typical emphasis and philosophy in the field parallel rendering focuses research on the hardware architecture's scalability; then one builds a machine big enough to render the desired data. Such an approach is neither sufficient for rendering today's largest data sets nor cost-effective for the increasing base of cluster users.

In this research project we have tightly integrate the use of level of detail with PC cluster-based rendering systems. As a product of this research, we have developed:

- $G L O D$, a widely deployable and publicly available level-of-detail system with a translucent, driver-level API supporting a variety of level of detail algorithms across a range of parallel rendering architectures.

- Level of detail algorithms for performing load management as well as load balancing of visualization clusters.

- Parallel algorithms for both level of detail construction and level of detail management.

- Level-of-detail approaches to data compression that are compatible with highperformance, highly parallel graphics hardware (GPUs)

- Debugging approaches leveraging visualization to explore large quantities of data within a modern parallel GPU

\section{Students and Collaborations}

This research project has also supported the development of a number of student researchers, including 3 PhDs (Kryzsztof Niski, Budirijanto Purnomo, and Yuan Chen, 1 MS (Nathaniel Duca), and 1 BS (also Nathaniel Duca). It enabled research discussions both remotely in person of the PI with DOE Researchers at LLNL: Randy Frank, Sean Ahearn, Mark Duchaineau, and Peter Lindstrom. These research collaborations have been so successful that the PI has actually joined the research team as an employee at LLNL. 


\section{Publications}

This research project has resulted in a number of technical publications, listed below. Many are available via the world-wide web at: http://www.cs.jhu.edu/ cohen/publications.html

In addition, the GLOD software is available at: http://www.cs.jhu.edu/ graphics/GLOD

1. Cohen, Jonathan D. and Dinesh Manocha. "Model Simplification for Interactive Visualization." Visualization Handbook. 13 pages. Eds. Chris Johnson and Chuck Hansen. Elsevier Butterworth-Heinemann. Chapter 20, pp. 393-410. 2005. (invited submission)

2. Niski, Krzysztof, and Jonathan D. Cohen. "Tile-based Level of Detail for the Parallel Age." IEEE Transactions on Visualization and Computer Graphics (Proceedings of IEEE Visualization 2007). 13 (6). pp. 1352-1359. 2007. (acceptance rate: 26\%) (accompanied by 5minute video)

3. Chen, Yuan, Jonathan D. Cohen, and Julian H. Krolik. "Similarity-Guided Streamline Placement with Error Evaluation." IEEE Transactions on Visualization and Computer Graphics (Proceedings of IEEE Visualization 2007). 13 (6). pp. 1448-1455. 2007. (acceptance rate: $26 \%)$

4. Niski, Krzysztof, Budirijanto Purnomo, and Jonathan D. Cohen. "Multi-grained Level of Detail Using a Hierarchical Seamless Texture Atlas." Proceedings of 2007 ACM Symposium on Interactive $3 D$ Graphics and Games. pp. 153-16o. (acceptance rate: 35\%) (accompanied by a 5 -minute video)

5. Sadowski, Ofri, Jonathan D. Cohen, Russell H. Taylor. "Projected Tetrahedra Revisited: A Barycentric Formulation Applied to Digital Radiograph Reconstruction Using Higher-Order Attenuation Functions." IEEE Transactions on Visualization and Computer Graphics. 12 (4). July 2006. pp. 461-473. (invited submission)

6. Chen, Yuan, Jonathan D. Cohen, and Subodh Kumar. "Visualization of Time-Varying Curvilinear Grids Using a 3D Warp Texture." 10th International Fall Workshop on Vision, Modeling, and Visualization 2005. pp. 281-288 and 514. (accompanied by a 4-minute video)

7. Sadowski, Ofri, Jonathan D. Cohen, and Russell H. Taylor. "Rendering Tetrahedral Meshes with Higher-Order Attenuation Functions for Digital Radiograph Reconstruction.” IEEE Visualization 2005. pp. 303-310. (accompanied by a 2-minute video) (nominated for Best Paper) (acceptance rate: 33\%)

8. Bilodeau, Jonathan, Budirijanto Purnomo, Jonathan D. Cohen, and Subodh Kumar. "Hardware-Compatible Vertex Compression Using Quantization and Simplification". ACM SIGGRAPH/Eurographics Symposium on Graphics Hardware 2005. pp. 53-61 and 117. (acceptance rate: 40\%)

9. Duca, Nathaniel, Chris Niski, Jonathan Bilodeau, Matthew Bolitho, Yuan Chan, and Jonathan Cohen. "A Relational Debugging Engine for the Graphics Pipeline. ACM Transactions on Graphics (Proceedings of SIGGRAPH 2005). pp. 453-463. (accompanied by a 5-minute video) (acceptance rate: 21\%)

10. Chhugani, Jatin, Budi Purnomo, Shankar Krishnan, Jonathan D. Cohen, Suresh Venkatasubramanian, David Johnson, Subodh Kumar. "vLOD: A System for High Fidelity Walkthroughs of Very Large Virtual Environments". IEEE Transactions on Visualization and Computer Graphics. 11 (1). January 2005. pp. 35-47.

11. Cohen, Jonathan, Dean Snyder, Donald Duncan, Jerrold Cooper, Subodh Kumar, Daniel Hahn, Yuan Chen, Budirijanto Purnomo, and John Graettinger. "iClay: Digitizing Cuneiform". Proceedings of $5^{\text {th }}$ International Eurographics Symposium on Virtual Reality, Archaeology, and Cultural Heritage. pp. 135-143. 2004. 
12. Purnomo, Budirijanto, Jonathan Cohen, and Subodh Kumar. "Seamless Texture Atlases" Proceedings of $2^{\text {nd }}$ Eurographics/ACM Symposium on Geometry Processing, pp. 67-76. 2004. (acceptance rate: 29\%)

13. Cohen, Jonathan, Dinesh Manocha, and Marc Olano. "Successive Mappings: An Approach to Polygonal Mesh Simplification with Guaranteed Error Bounds." International Journal of Computational Geometry and Applications. 13 (1). February 2003. pp. 61-94.

14. Kumar, Subodh, Dean Snyder, Donald Duncan, Jonathan Cohen, and Jerry Cooper. "Digital Preservation of Ancient Cuneiform Tablets Using 3D-Scanning." Proceedings of Fourth International Conference on 3 D Digital Imaging and Modeling. pp. 326-333. 2003. (selected for cover image) (acceptance rate: 40\%)

15. Chhugani, Jatin, Sudhir Vishwanath, Jonathan Cohen, and Subodh Kumar. ISOSLIDER: A System for Interactive Exploration of Isosurfaces. Proceedings of the Joint Eurographics IEEE TCVG Symposium on Visualization (VisSym 2003). pp. 259-266. 2003. (acceptance rate: $48 \%$ )

16. Williams, Nathaniel, Michael Kelly, Brendon Schubert, David Luebke, and Jonathan Cohen. "Perceptually Guided Simplification of Lit, Textured Meshes." Proceedings of 2003 ACM Symposium on Interactive 3D Graphics. pp. 113-121. (selected for cover image) (acceptance rate: $26 \%$ )

17. Hahn, Daniel V., Donald D. Duncan, Kevin C. Baldwin, Jonathan D. Cohen, and Budirijanto Purnomo. "Digital Hammurabi: Design and Development of a 3D Scanner for Cuneiform Tablets." Proceedings of IS\&T/SPIE $18^{\text {th }}$ Annual Symposium Electronic Imaging and Science and Technology. 6056. pp. 130-141. 2006.

18. Duca, Nathaniel and Jonathan Cohen. "Building a Graphics Debugger." ACM SIGGRAPH Technical Sketches. accepted. 1 page.

19. Niski, Krzysztof and Jonathan Cohen. "GPU-based LOD of Genus-Zero Models." submitted to ACM SIGGRAPH Technical Sketches. 1 page.

20. Chen, Yuan, Jonathan D. Cohen, and Subodh Kumar. "Visualization of Time-Varying Scalar Fields on Curvilinear Grids Using a 3D Warp Texture." submitted to ACM SIGGRAPH Technical Sketches. 1 page.

21. Chen, Yuan, Jonathan D. Cohen, and Subodh Kumar. "On the Visualization of Time-Varying Structured Grids Using a 3D Warp Texture.” IEEE Visualization 2004 Poster and Interactive Demo. pp. 107-108. (received Best Poster Award out of 35 accepted posters).

22. Cohen, Jonathan D., David Luebke, Nathaniel Duca, Brenden Schubert, and Chris Niski. "GLOD: A Minimal Interface for Geometric Level of Detail". 10 pages. Johns Hopkins University Graphics Lab Technical Report. 2004.

23. Cohen, Jonathan D., David Luebke, Nathaniel Duca, and Brenden Schubert. "GLOD: A Geometric Level of Detail System at the OpenGL API Level.” IEEE Visualization 2003 Poster (2 pages). (received Best Poster Award out of 32 accepted posters).

24. Cohen, Jonathan D., David Luebke, Nathaniel Duca, and Brenden Schubert. "GLOD: A Driver-Level Interface for Geometric Level of Detail.” SIGGRAPH 2003 Technical Sketch.

25. Cohen, Jonathan D., David Luebke, Nathaniel Duca, and Brenden Schubert. "GLOD: Level of Detail for the Masses.” Johns Hopkins University Graphics Lab Technical Report. 2003. 